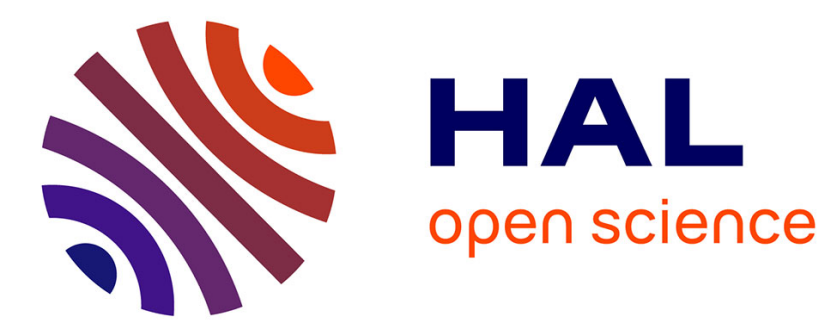

\title{
Ptychography in anisotropic media
}

Patrick Ferrand, Marc Allain, Virginie Chamard

\section{To cite this version:}

Patrick Ferrand, Marc Allain, Virginie Chamard. Ptychography in anisotropic media. Optics Letters, 2015, 40 (22), pp.5144-5147. 10.1364/OL.40.005144 . hal-01213942

\section{HAL Id: hal-01213942 \\ https://hal.science/hal-01213942}

Submitted on 16 Oct 2015

HAL is a multi-disciplinary open access archive for the deposit and dissemination of scientific research documents, whether they are published or not. The documents may come from teaching and research institutions in France or abroad, or from public or private research centers.
L'archive ouverte pluridisciplinaire HAL, est destinée au dépôt et à la diffusion de documents scientifiques de niveau recherche, publiés ou non, émanant des établissements d'enseignement et de recherche français ou étrangers, des laboratoires publics ou privés. 


\title{
Ptychography in anisotropic media
}

\author{
Patrick Ferrand ${ }^{1 *}$, Marc Allain ${ }^{1}$, And Virginie Chamard ${ }^{1}$ \\ ${ }^{1}$ Aix-Marseille Université, CNRS, Institut Fresnel, Centrale Marseille, UMR 7249, Institut Fresnel, F-13013 Marseille, France \\ ${ }^{*}$ Corresponding author: patrick.ferrand@fresnel.fr
}

Compiled October 12, 2015

\begin{abstract}
Ptychography is described in the context of polarized light probing anisotropic specimen, i.e., showing properties of birefringence and/or diattenuation. We establish an optimization strategy using a vectorial formalism. A measurement scheme using a set of linearly polarized probes and linear polarization analyzers is proposed, allowing to retrieve the full anisotropy map of the specimen. (- 2015 Optical Society of America
\end{abstract}

OCIS codes: 110.5405 Polarimetric imaging; 110.3010 Image reconstruction techniques; 100.5070 Phase retrieval; 120.5410 Polarimetry; 260.5430 Polarization; 340.0340 X-ray optics

http://dx.doi.org/xxx

Ptychography is an imaging technique aiming at reconstructing numerically the transmission properties of an object (amplitude, phase). It exploits a set of intensity diffraction patterns recorded for different positions of a spatially-structured illumination probe on the object [1]. In the context of optical microscopy, it is becoming a new competitive method mostly thanks to its capability to image quantitatively the phase shift introduced by the sample. Beyond the possibility which is now offered to see transparent specimen, measuring the phase brings a valuable information, like e. g., in biology for the quantification of the mass of cells [2-4] or chromosomes [5]. In spite of this progress, ptychography has been only developed in a scalar formalism, an approximation that restricts its application to isotropic materials. Though, many systems, because of their structural molecular anisotropy, present strong optical birefringence properties, as it is the case for biological cell components (actine [6], microtubules [7]), biological tissues (collagen [8]), or biomineral systems [9]. There is therefore a strong need for the development of a vectorial formalism, that would allow a complete optical characterization of these anisotropic materials. Moreover, addressing anisotropy is not limited to the optical waves. In electron [10] and x-ray [11-13] microscopy, where much progress in high resolution imaging has been accomplished with ptychography using a scalar formalism, exploiting the specific interaction between a polarized beam and materials showing anisotropic electronic properties such as dichroism [14] requires a generic vectorial formalism, too.

In this letter, we revisit the ptychography problem using a vectorial formalism and establish a criterium to be minimized, in order to reconstruct the anisotropy properties of the object. A measurement scheme using a set of linearly polarized probes and polarization analyzers is proposed, allowing the retrieval of the full anisotropic properties of the specimen without indetermination.

Ptychography relies on the assumption that a probe interacts with a specimen in a multiplicative way [15]. For a full vectorial description, it is convenient to use the formalism introduced by Jones [16], so that the $k$-th probe $\mathbf{p}_{k}$, e.g., the $k$-th polarization state, centered at the $j$-th position $\mathbf{r}_{j}$, must be written as a complex vector

$$
\mathbf{p}_{j k}(\mathbf{r})=\mathbf{p}_{k}\left(\mathbf{r}-\mathbf{r}_{j}\right)=\left[\begin{array}{c}
p_{j k ; x}(\mathbf{r}) \\
p_{j k ; y}(\mathbf{r})
\end{array}\right],
$$

where the two complex components $p_{j k ; x}(\mathbf{r})$ and $p_{j k ; y}(\mathbf{r})$ refer to the $x$ and $y$ transverse directions, respectively. The specimen transmission properties have to be described at any position $\mathbf{r}$ by a so-called Jones complex matrix

$$
\mathbf{O}(\mathbf{r})=\left[\begin{array}{ll}
\rho_{x x}(\mathbf{r}) & \rho_{y x}(\mathbf{r}) \\
\rho_{x y}(\mathbf{r}) & \rho_{y y}(\mathbf{r})
\end{array}\right],
$$

where, in general $\rho_{y x}(\mathbf{r}) \neq \rho_{x y}(\mathbf{r})$ [16]. With these notations, the vectorial exit field $\boldsymbol{\psi}_{j k}(\mathbf{r})$ of the ptychography problem is simply given by the matrix multiplication

$$
\boldsymbol{\psi}_{j k}(\mathbf{r})=\mathbf{O}(\mathbf{r}) \mathbf{p}_{j k}(\mathbf{r}) .
$$

Thus, solving the ptychography problem in an anisotropic medium aims at retrieving the 4-element Jones matrix $\mathbf{O}(\mathbf{r})$ at any point $\mathbf{r}$ or, equivalently, at retrieving the four maps $\rho_{x x}(\mathbf{r})$, $\rho_{y x}(\mathbf{r}), \rho_{x y}(\mathbf{r})$, and $\rho_{y y}(\mathbf{r})$. In the following, we will refer to this four-map set as the Jones map of the object.

Now that the interaction between the probe and the specimen is formally established, the equations of the direct ptychography problem can be derived and the fitting strategies for reconstructing the object can be formulated. For the convenience of these derivations, we will, temporarily and without loss of generality, adopt a different formalism, so that Eq. 3 becomes

$$
\psi_{j k}(\mathbf{r})=\mathbf{P}_{j k}(\mathbf{r}) \boldsymbol{\rho}(\mathbf{r}),
$$

where we have built up, out of $\mathbf{O}(\mathbf{r})$ and $\mathbf{p}_{j k}(\mathbf{r})$, the two quantities

$$
\mathbf{P}_{j k}(\mathbf{r})=\left[\begin{array}{cccc}
p_{j k ; x}(\mathbf{r}) & 0 & p_{j k ; y}(\mathbf{r}) & 0 \\
0 & p_{j k ; y}(\mathbf{r}) & 0 & p_{j k ; x}(\mathbf{r})
\end{array}\right]
$$


and

$$
\boldsymbol{\rho}(\mathbf{r})=\left[\begin{array}{c}
\rho_{x x}(\mathbf{r}) \\
\rho_{y y}(\mathbf{r}) \\
\rho_{y x}(\mathbf{r}) \\
\rho_{x y}(\mathbf{r})
\end{array}\right] .
$$

The far field generated in the Fraunhoffer approximation by this exit-field reads $\boldsymbol{\Psi}_{j k}(\mathbf{q})=\mathbf{F} \boldsymbol{\psi}_{j k}(\mathbf{r})$, where $\mathbf{q}$ is the reciprocalspace coordinate, with

$$
\mathbf{F}=\left[\begin{array}{cc}
\mathcal{F} & 0 \\
0 & \mathcal{F}
\end{array}\right]
$$

where $\mathcal{F}$ is the 2D Fourier transform. After a polarization analysis, the vectorial field $\boldsymbol{\Psi}_{j k}$ is reduced to a scalar complex amplitude to

$$
\xi_{j k l}(\mathbf{q})=\mathbf{h}_{l}^{T} \mathbf{\Psi}_{j k}(\mathbf{q}),
$$

where $\mathbf{h}_{l}$ is the corresponding operator for the $l$-th analysis filter, and $T$ is the transpose operator. For instance, one can show easily that the operator corresponding to a linear polarizer oriented at $\alpha$ with respect to the $x$ direction is

$$
\mathbf{h}_{\alpha}=\left[\begin{array}{c}
\cos \alpha \\
\sin \alpha
\end{array}\right] .
$$

Finally, the intensity $y_{j k l}(\mathbf{q})$ collected in the detection plane reads (in average)

$$
u_{j k l}(\mathbf{q}) \equiv\left\langle y_{j k l}(\mathbf{q})\right\rangle=\left|\xi_{j k l}(\mathbf{q})\right|^{2}+\epsilon_{k l}(\mathbf{q}),
$$

with $\epsilon_{k l}$ the expected contribution of the background component to the intensity.

Because Eq. 9 gives the formal relationship between the measurement $y_{j k l}$ and the unknown object $\rho$, it gives us the opportunity to define a least-square estimate of the unknown object via the minimization of the following criterion [17]

$$
\mathcal{L}(\boldsymbol{\rho})=\sum_{j} \sum_{k, l}\left|y_{j k l}^{1 / 2}-u_{j k l}^{1 / 2}\right|^{2}=\sum_{j} \mathcal{L}_{j}(\boldsymbol{\rho}) .
$$

Following the same "spirit" as the well-known ptychographical iterative engine (PIE) algorithm established in a scalar formalism [18], the vectorial PIE (vPIE) needs to compute the fourcomponent gradient of $\mathcal{L}_{j}$, that reads

$$
\frac{\partial \mathcal{L}_{j}}{\partial \rho}=-\sum_{k, l} \frac{\partial u_{j k l}}{\partial \rho}\left[y_{j k l}^{1 / 2}-u_{j k l}^{1 / 2}\right] u_{j k l}^{-1 / 2},
$$

leading to

$$
\frac{\partial \mathcal{L}_{j}}{\partial \boldsymbol{\rho}}=-2 \sum_{k} \mathbf{P}_{j k}^{\dagger} \mathbf{F}^{\dagger} \sum_{l} \mathbf{h}_{l}^{\star}\left(\xi_{j k l}^{\prime}-\xi_{j k l}\right),
$$

where $\xi_{j k l}^{\prime}$ is the updated scalar far field, meaning that the modulus is replaced by the square root of the measured intensity, $\xi_{j k l}^{\prime}=\sqrt{y_{j k l} / u_{j k l}} \xi_{j k l}$, and where $\star$ and + denote the "complex conjugate" and "transposed complex conjugate" operators, respectively.

Finally, similarly to the scalar PIE, the optimization algorithm is now applied to the four-component guess of the object, i.e. to the Jones map, that is updated for each step of the probe position according to

$$
\boldsymbol{\rho}^{(j+1)}=\boldsymbol{\rho}^{(j)}-\beta \mathbf{D}_{j} \frac{\partial \mathcal{L}_{j}}{\partial \boldsymbol{\rho}},
$$

where $\beta>0$ is the step size of the update, and $\mathbf{D}_{j}$ is a $4 \times 4$ diagonal matrix, with diagonal values given by $D_{j, 1}=D_{j, 4}=$ $1 / \max _{\mathbf{r}}\left(\sum_{k}\left|p_{j k ; x}\right|^{2}\right)$ and $D_{j, 2}=D_{j, 3}=1 / \max _{\mathbf{r}}\left(\sum_{k}\left|p_{j k ; y}\right|^{2}\right)$.
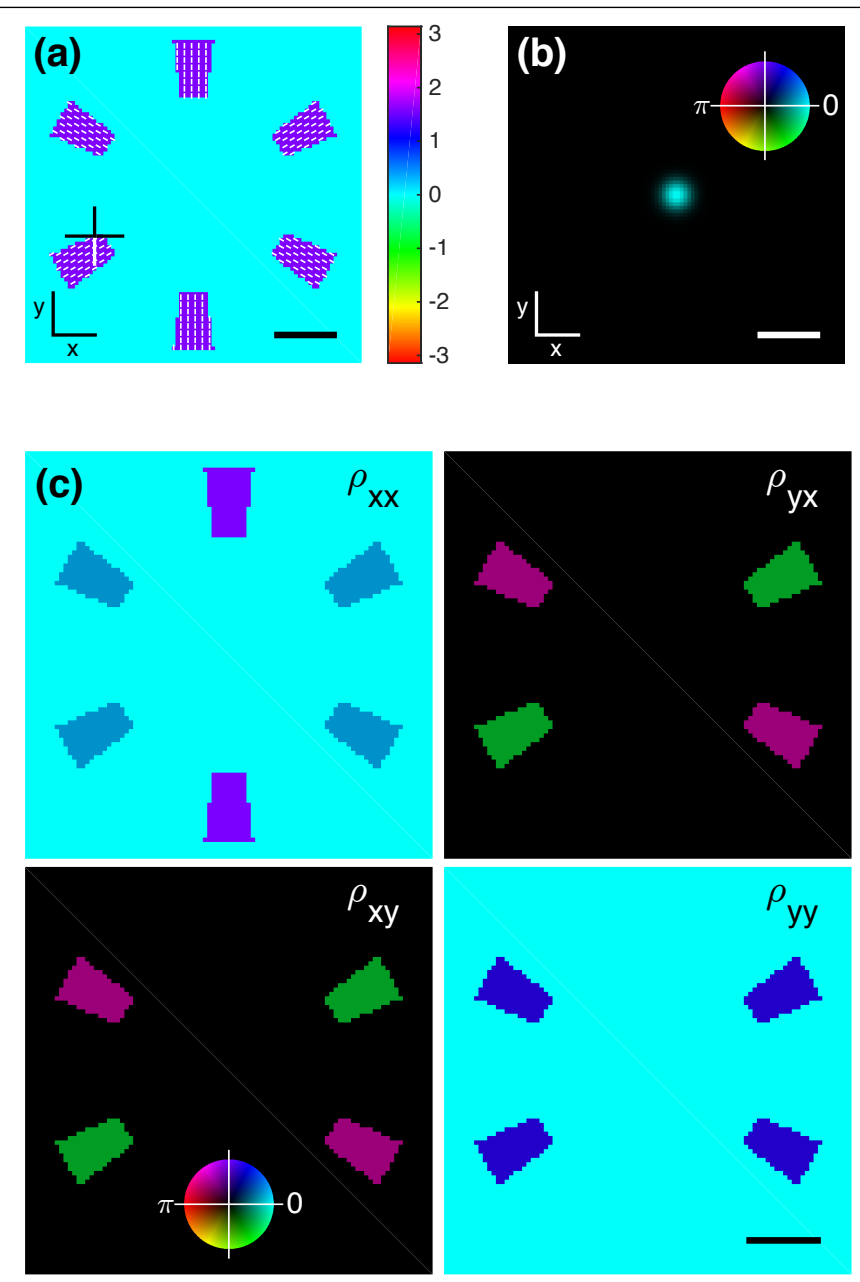

Fig. 1. (a) Simulated object. Colorscale indicates the retardance in radians. Superimposed white sticks show the orientation of the fast axis for birefringent area. (b) Complex amplitude of the probe. The inset shows the complex value color coding, with phase encoded as hue and modulus as brightness. (c) Jones map of the simulated object. All scale bars are $20 \mu \mathrm{m}$.

Retrieving the full anisotropy properties of any object $\mathbf{O}$ requires that the measurement involves the appropriate set of probes and analysis filters. A naive approach would consist in addressing independently each term of the Jones map, i.e., probe and analysis polarized along the $x$ direction in order to address $\rho_{x x}$, probe along $x$ and analysis along $y$ to address $\rho_{x y}$, etc. However, since each map of $\rho_{x x}, \rho_{x y}, \rho_{y x}, \rho_{y y}$ will be retrieved, each one with a constant phase indetermination, this method would fail in reconstructing the phase relationship between the four elements of the Jones map. Therefore we propose the following strategy for choosing the probes and analyzers. Concerning 


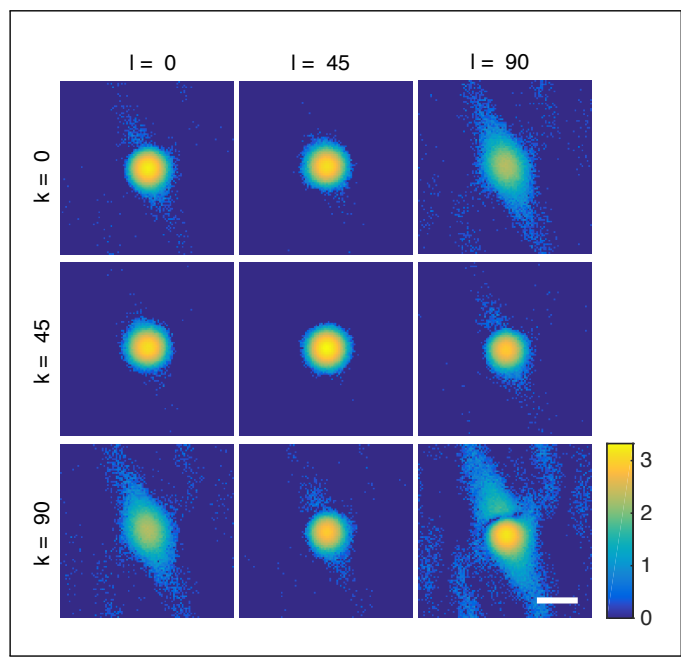

Fig. 2. Nine intensity patterns (log of intensity) as recorded for all $k l$ combinations, when the probe is centered at the point indicated by the cross in Fig. 1a. Color scale refer to all patterns. Scale bar is $1 \mu \mathrm{m}^{-1}$.

the probes and more specifically for linear polarization states, the sensitivity to the anisotropy properties requires that their polarization angle is chosen as far as possible from the directions defined by the neutral axes of the material. Without a priori knowledge of these axes in the investigated object, a common strategy to avoid pathological cases in polarimetry consists in using three different polarization angles [19]. Therefore, we propose to use a set of three linearly-polarized probes $\mathbf{P}_{j k}$, where $k \in\{0,45,90\}$ denotes the orientation in degrees of the linear polarization of the field in the $x y$ plane. Concerning the polarization analysis, without a priori knowledge of the kind of polarization state (linear, circular, etc.) to be analyzed, we also propose to use three linear analysis filters, for instance along the same directions, $\mathbf{h}_{l}$, with $l \in\{0,45,90\}$. Thus the vPIE exploits a set of nine intensity patterns $u_{j k l}(\mathbf{q})$, corresponding to all the combinations of probes and analyzers, such high number of measurements aiming at solving unambiguously the whole set of unknowns, namely the four complex components of $\mathbf{O}(\mathbf{r})$. With these sets of probes and analyzers, the update of $\mathbf{O}$ as proposed by Eq. 13 writes explicitly, for instance for the $\rho_{x x}$ component,

$$
\begin{aligned}
\rho_{x x}^{(j+1)}= & \rho_{x x}^{(j)}+\frac{2 \beta}{\max _{\mathbf{r}}\left(\left|p_{j, 0, x}\right|^{2}+\left|p_{j, 45, x}\right|^{2}\right)} \\
\times & {\left[p_{j, 0, x}^{*}\left(\Delta \psi_{j, 0,0}+\frac{1}{\sqrt{2}} \Delta \psi_{j, 0,45}\right)\right.} \\
& \left.+p_{j, 45, x}^{*}\left(\Delta \psi_{j, 45,0}+\frac{1}{\sqrt{2}} \Delta \psi_{j, 45,45}\right)\right],
\end{aligned}
$$

with $\Delta \psi_{j k l}=\mathcal{F}^{-1}\left(\xi_{j k l}^{\prime}-\xi_{j k l}\right)$.

In order to test the validity of our approach, the vPIE has been run on a set of simulated data. We have considered a set of six distant birefringent objects of retardance $\pi / 2$ regularly arranged on a ring, having their fast axis oriented radially (Fig. 1a), surrounded by an isotropic medium, chosen so that the objects do not generate any phase step for an electric field polarized along the direction of the fast axis. Although different in terms of polarization orientations, the three probes have the same two-dimensional gaussian profile (radius $3.5 \mu \mathrm{m}$ ) illustrated in Fig. 1b, and a wavelength of $0.5 \mu \mathrm{m}$. The corresponding
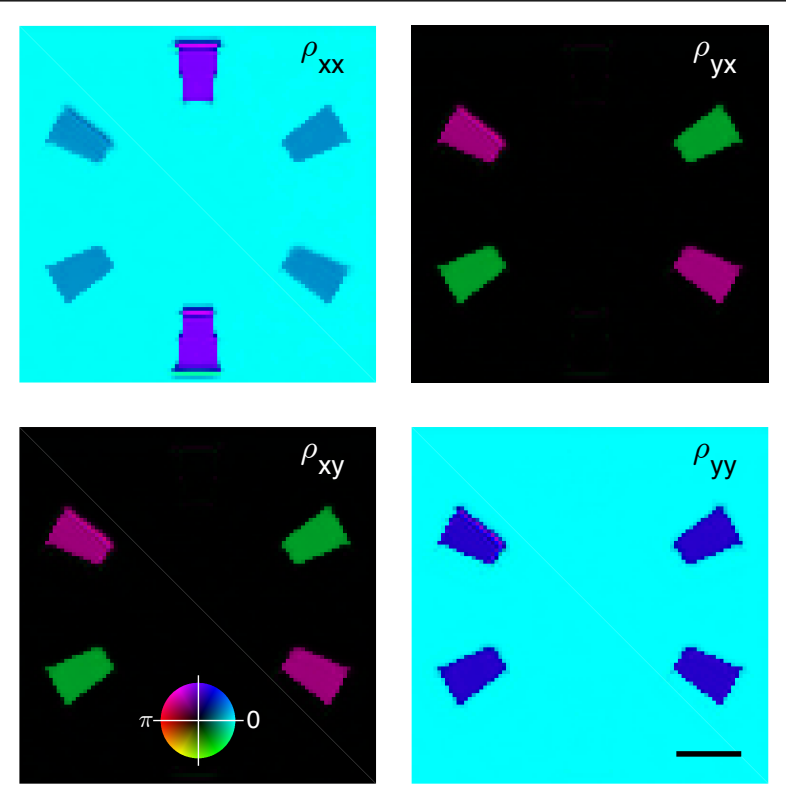

Fig. 3. Reconstructed Jones map of the object. The inset shows the complex value color coding, with phase encoded as hue and modulus as brightness. Scale bar is $20 \mu \mathrm{m}$.

Jones map of this object is presented in Fig. 1c, showing clearly different values for the different areas, due to their different orientations. In addition, one can notice that $\rho_{y x}(\mathbf{r})=\rho_{y x}(\mathbf{r})$, which is the signature that the object is made of single-layer birefringent regions (in opposition to stacks) [16]. The probes were scanned over the object in steps of $2.5 \mu \mathrm{m}$, generating at 2,500 positions the nine intensity patterns as recorded by a $100 \times 100$ pixels (pixel size $8 \mathrm{~mm}$ ) camera placed in the Fourier plane of a lens of focal length $20 \mathrm{~mm}$. A Poisson random number generator was used in order to mimic the shot noise of the signal. Total number of counts over a frame were typically 200,000. An example of set of intensity patterns is shown on Fig. 2.

The vPIE was run using a random distribution of modulus and phase for all components of $\mathbf{O}(\mathbf{r})$ as starting guess. The Jones map of the object, as reconstructed after 100 full-scan itera-

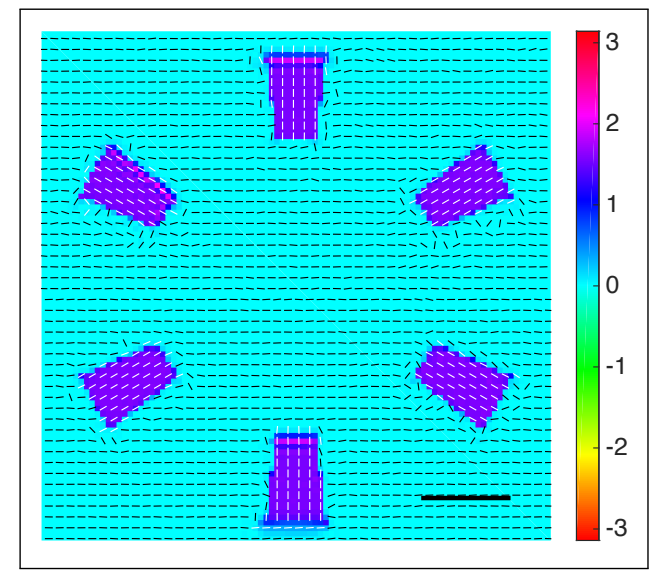

Fig. 4. Retrieved retardance $\varphi$ (colorscale in radians) and fast axis orientation $\theta$ (represented by superimposed sticks). Scale bar is $20 \mu \mathrm{m}$. 


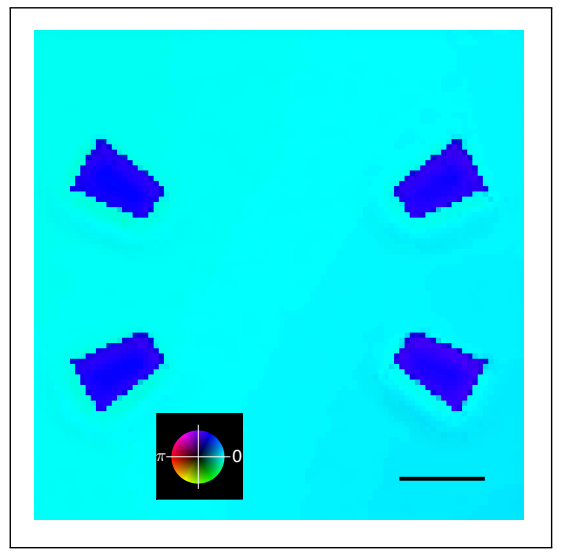

Fig. 5. Complex amplitude of the object as retrieved from a scalar ptychography measurement. Scale bar is $20 \mu \mathrm{m}$.

tions with $\beta=0.5$, is shown in Fig. 3, after subtraction of a global phase offset to all four components of $\mathbf{O}(\mathbf{r})$, in order to ensure an arbitrary phase of zero for $\rho_{x x}$ in the surrounding medium. This reconstructed Jones map is in excellent agreement with the simulated one of Fig. 1c. The smoothing of the edges was found to be a consequence of the simulated shot noise, which operates a truncation of the highest $q$ values in the intensity patterns (Fig. 2) [17]. Since the specimen was made of single layer object, we could produce a more comprehensive picture of the object properties, by retrieving independently the values of retardance $\varphi(\mathbf{r})$ and fast axis orientation $\theta(\mathbf{r})$. Mathematically, this was done by solving at every point $\mathbf{r}$ the basic matrix equation

$$
\mathbf{O}=\mathbf{R}(-\theta) \mathbf{W}(\varphi) \mathbf{R}(\theta),
$$

where

$$
\mathbf{R}(\theta)=\left[\begin{array}{cc}
\cos \theta & \sin \theta \\
-\sin \theta & \cos \theta
\end{array}\right] \text { and } \mathbf{W}(\varphi)=\left[\begin{array}{cc}
1 & 0 \\
0 & \exp (i \varphi)
\end{array}\right] .
$$

The result is shown in Fig. 4. The retrieved retardance value of $\pi / 2$ for all objects and the radial orientations reproduce very well the initial model of Fig. 1a.

For comparison purpose, the simulated dataset was also processed by the scalar PIE, where we considered as intensity

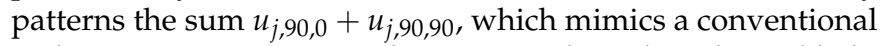
scalar measurements carried out using a linearly-polarized light source (here along the $y$ direction) and no polarization analysis. The scalar object, as reconstructed using the same number of iterations and $\beta$ value, is shown in Fig. 5. Predictively, the two blocks located at the top and the bottom of the object are no longer visible, since they have been designed not to produce any phase step in the transmitted field for this polarization orientation. Although the retrieved object looks similar to $\rho_{y y}$ (Fig. 1c), the obtained complex values are significantly different. For instance, within the upper-right block, the modulus is $0.8 \mathrm{vs} .1$ for $\rho_{y y}$ at the same point in Fig. 5 , and the phase is 1.27 vs. 1.15 . In addition, the reconstructed surrounding medium exhibits some ghost artefacts. These observations confirm the relevance of the vPIE, as soon as the sample possesses some birefringence properties.

We have run vPIE successfully on a large variety of anisotropic objects. Although only shown here in the case of birefringent objects, we have obtained excellent results for object showing properties of diattenuation, i.e., anisotropic absorbance.
For all investigated cases, object reconstructions converge typically in 10-100 iterations towards a solution, except for the very well known pathological case of an homogeneous medium, for which the distinction between $\boldsymbol{\rho}(\mathbf{r})$ vs. $\boldsymbol{\rho}^{*}(-\mathbf{r})$ cannot exploit on the spatial diversity of ptychography.

In conclusion, we have revisited the ptychography problem using a vectorial formalism. A criterion has been derived and a measurement scheme using a set of linearly polarized probes and polarization analyzers has been proposed. Simulations show that the full anisotropic properties of the specimen can be effectively retrieved without indetermination. This work opens new perspectives for the exhaustive investigation of anisotropic materials by means of ptychography.

\section{FUNDING INFORMATION}

Agence Nationale de la Recherche (ANR) (ANR-11-BS10-0005).

\section{ACKNOWLEDGMENTS}

The authors acknowledge Julien Duboisset for fruitful discussions.

\section{REFERENCES}

1. H. M. L. Faulkner and J. M. Rodenburg, Phys. Rev. Lett. 93, 023903 (2004).

2. M. Mir, Z. Wang, Z. Shen, M. Bednarz, R. Bashir, I. Golding, S. G. Prasanth, and G. Popescu, Proc. Nat. Acad. Sci. 108, 13124-13129 (2011).

3. J. Marrison, L. Räty, P. Marriott, and P. O'Toole, Sci. Rep. 3, 2369 (2013).

4. T. A. Zangle and M. A. Teitell, Nat. Meth. 11, 1221-1228 (2014).

5. L. Shemilt, E. Verbanis, J. Schwenke, A. K. Estandarte, G. Xiong, R. Harder, N. Parmar, M. Yusuf, F. Zhang, and I. K. Robinson, Biophys. J. 108, 706-713 (2015).

6. K. Katoh, G. Langford, K. Hammar, P. J. S. Smith, and R. Oldenbourg, Biol. Bull. 193, 219-220 (1997).

7. R. Oldenbourg, E. D. Salmon, and P. T. Tran, Biophys. J. 74, 645-654 (1998).

8. M. Wolman and F. H. Kasten, Histochem. 85, 41-49 (1986).

9. L. Beaufort, Micropaleontology 51, 289-297 (2005).

10. M. J. Humphry, B. Kraus, A. C. Hurst, A. M. Maiden, and J. M. Rodenburg, Nat. Commun. 3, 730 (2012).

11. J. M. Rodenburg, A. C. Hurst, A. G. Cullis, B. R. Dobson, F. Pfeiffer, O. Bunk, C. David, K. Jefimovs, and I. Johnson, Phys. Rev. Lett. 98, 034801 (2007).

12. M. Dierolf, A. Menzel, P. Thibault, P. Schneider, C. M. Kewish, R. Wepf, O. Bunk, and F. Pfeiffer, Nature 467, 436-439 (2010).

13. V. Chamard, M. Allain, P. Godard, A. Talneau, G. Patriarche, and M. Burghammer, Sci. Rep. 5, 9827 (2015).

14. A. Tripathi, J. Mohanty, S. H. Dietze, O. G. Shpyrko, E. Shipton, E. E. Fullerton, S. S. Kim, and I. McNulty, Proc. Nat. Acad. Sci. USA 108, 13393-13398 (2011).

15. J. M. Rodenburg and R. H. T. Bates, "The theory of super-resolution electron microscopy via Wigner-distribution deconvolution," Philos. T. Roy. Soc. A 339, 521-553 (1992).

16. R. C. Jones, "A new calculus formalism for the treatment of optical systems. I. Description and discussion of the method," J. Opt. Soc. Amer. 31, 488-493 (1941).

17. P. Godard, M. Allain, V. Chamard, and J. Rodenburg, "Noise models for low counting rate coherent diffraction imaging," Opt. Express 20, 25914-25934 (2012).

18. J. M. Rodenburg and H. M. L. Faulkner, "A phase retrieval algorithm for shifting illumination," Appl. Phys. Lett. 85, 4795 (2004).

19. E. Collett, Polarized light. Fundamentals and applications (Marcel Dekker, New York, 1993). 